\title{
The Interval Stability of an Electricity Market Model
}

\author{
Weijuan Wang, ${ }^{1}$ Zhanhui Lu, ${ }^{1}$ and Quanxin $\mathrm{Zhu}^{2}$ \\ ${ }^{1}$ School of Mathematics and Physical Science, North China Electric Power University, Beijing 102206, China \\ ${ }^{2}$ School of Mathematical Sciences and Institute of Finance and Statistics, Nanjing Normal University, Nanjing 210023, China \\ Correspondence should be addressed to Quanxin Zhu; zqx22@126.com
}

Received 29 October 2014; Accepted 12 December 2014; Published 30 December 2014

Academic Editor: Xinkai Chen

Copyright (C) 2014 Weijuan Wang et al. This is an open access article distributed under the Creative Commons Attribution License, which permits unrestricted use, distribution, and reproduction in any medium, provided the original work is properly cited.

\begin{abstract}
Combined with the electric power market dynamic model put forward by Alvarado, an interval model of electricity markets is established and investigated in this paper pertaining to the range of demand elasticity with suppliers and consumers. The stability of an electricity market framework with demand elasticity interval is analyzed. The conclusions characterizing the interval model provided are derived by constructing a suitable Lyapunov function and using the theory of interval dynamical system in differential equations and matrix inequality theory and so forth. Applying the corollary obtained can judge the system stability by available data about demand elasticity. The obtained results are validated and illustrated by a case example.
\end{abstract}

\section{Introduction}

Power system is the physical basis for electricity market and electricity market is the operating mode of power system. The economic stability of electricity market and the physical stability of power system are linked and affect each other. In 1990, Beavis and Dobbs began to study the economic system stability [1]. For twenty years now, the research on the dynamic evolution and stable behavior of economy and management system has been arousing increasing attention in many fields such as mathematical economics and system science. Particularly, the stability analysis of economic system branch is emphasized in economic area, which is of difficulty and great significance as well.

While the study of electricity market stability begins to perk up in recent years [2-10], power resources are allocated reasonably by market mechanism in electricity market, and the research on the electricity market stability is of very vital significance for regulating the market supply and demand. Based on the electricity market dynamic model proposed by Alvarado [2], a new electricity market model was provided within the dynamic market framework on the consideration of factors such as electrical energy unbalance and dynamic power system properties after cooperation with Meng and others [3-5]. The numerical eigenvalue method was adopted to study the electricity market stability in all of the dynamic models above.
In [11], a kind of promotion for the model of Alvarado was made in respect of market clearing time and price signal delay, and the stability effect pertaining to system control and communication was discussed. With the dynamic market model, the role that futures markets may play on the clearing prices and on altering the volatility and potential instability of real-time prices and generator output was examined by Watts and Alvarado [12]. Reference [13] adopted a novel approach for system controllers characterizing Nordic power market, and it was concluded that electricity prices most of the time have increased in stability and decreased in volatility when the market has expanded and the competition degree has added. In [14], an appropriate modeling of system controllers is presented to account for the effects of power system controllers on the stability of power dispatch and electricity market prices better. For the market dynamic model put forward by Alvarado, a series of sufficient conditions are provided theoretically to determine the stability of power market in [15]. With all the above, the involved discussion about stability is derived from the Lyapunov asymptotic stability of equilibrium in the deterministic differential equation theory.

If the parameters are estimated using confidence intervals within a system, then the system is an interval dynamic system. In fact, due to the influence of the related factors such as measurement error, technical level, and other external interference, the accurate values of some parameters cannot be 
obtained. Therefore, interval theory should be introduced to describe problems more appropriately in ecology, economics, and other fields. So far, there have existed some discussions on power system research by using the interval method [16-18]. Due to the influence of dynamic factors in electricity market such as economic growth, electricity consumption, electricity price, supply, and demand, the demand elasticity of suppliers and consumers continues to change within a certain range instead of constants. So it is necessary to describe demand elasticity by interval. Alternatively, the theory of interval dynamical system is rarely applied to study the stability of the electric power market.

Adopting the method of interval dynamical system, this paper presents a novel interval model for the electricity market based on the deterministic dynamic model put forward by Alvarado. Embarking from the stability theory, this paper analyzes the influence of demand elasticity change on the system stability. By means of constructing a suitable Lyapunov function and using the theory of interval dynamical system in differential equations and matrix inequality theory, the stability of this interval model is investigated theoretically and some sufficient conditions of interval stability are given in our theorem and corollary. It is shown in the results that we can provide the stability scopes of demand elasticity for suppliers and consumers and judge the system stability by available data. Finally, an electricity market specific example is given and analyzed to illustrate the obtained results.

\section{The Establishment of the Electricity Market Interval Model}

2.1. Electricity Market Model Proposed by Alvarado. Let cost functions for generators and utility functions for consumers be quadratic functions. With $m$-supplier and $n$-consumer, when the market price $\lambda$ is greater than the marginal production cost $\lambda_{g i}$ in electricity market, suppliers will expand production until the marginal cost of production equals the price. The rate of expansion is proportional to the difference between the price observed and the actual production cost. The speed with which the generation power output $P_{g i}$ of supplier $i$ can respond is supplier dependent [2]. The above yields the following differential equation to describe the dynamic behavior of electricity market for supplier $i$ :

$$
\tau_{g i} \dot{P}_{g i}=\lambda-b_{g i}-c_{g i} P_{g i} \quad(i=1,2, \ldots, m),
$$

where $P_{g i}$ is the generation power output, the speed of which is denoted by a time constant $\tau_{g i}, \lambda$ is the market price at any given time, $b_{g i}+c_{g i} P_{g i}$ is the marginal cost, $c_{g i}$ is the demand elasticity, and $b_{g i}$ is the linear cost coefficient.

As for consumer $j$, the equation describing electricity market behavior is

$$
\tau_{d j} \dot{P}_{d j}=-\lambda+b_{d j}+c_{d j} P_{d j} \quad(j=1,2, \ldots, n),
$$

where $P_{d j}$ is the consumption demand of electricity, $\tau_{d j}$ is the expansion speed of consumption demand, $b_{d j}+c_{d j} P_{d j}$ is the marginal benefit, $c_{d j}$ is the demand elasticity, and $b_{d j}$ is the linear cost coefficient.
In addition, the following equation is considered:

$$
\sum_{i=1}^{m} P_{g i}=\sum_{j=1}^{n} P_{d j}
$$

Considering the transmission congestion of power market, using flow distribution factors, a single congested condition can be represented as a scalar additional equality constraint:

$$
\begin{aligned}
& s_{g 1} P_{g 1}+s_{g 2} P_{g 2}+\cdots+s_{g m} P_{g m} \\
& \quad+s_{d 1} P_{d 1}+s_{d 2} P_{d 2}+\cdots+s_{d n} P_{d n}=h_{1} .
\end{aligned}
$$

With $n_{S}$ congestion conditions, the complete model is

$$
\left[\begin{array}{cccccc}
1 & \cdots & 1 & -1 & \cdots & -1 \\
s_{g 11} & \cdots & s_{g 1 m} & s_{d 11} & \cdots & s_{d 1 n} \\
\vdots & & \vdots & \vdots & & \vdots \\
s_{g n_{S} 1} & \cdots & s_{g n_{S} m} & s_{d n_{S} 1} & \cdots & s_{d n_{s} n}
\end{array}\right]\left[\begin{array}{c}
P_{g 1} \\
\vdots \\
P_{g m} \\
P_{d 1} \\
\vdots \\
P_{d n}
\end{array}\right]=\left[\begin{array}{c}
0 \\
h_{1} \\
h_{2} \\
\vdots \\
h_{n_{S}}
\end{array}\right]
$$

Congestion equality constraints are algebraic. Thus, the analysis of the equilibrium as well as the stability properties of the congested market required the consideration of a DAE set of equations with multiple algebraic constraints. Of necessity, the congestion problem requires that higher-order nontrivial systems be considered. The issue is resolved by introducing shadow prices (Lagrange multipliers $\mu$ ) which play the role of congestion prices. If this is done, the complete dynamic equations for the congested $m$-supplier and $n$-consumer with $n_{S}$ active algebraic congestion conditions can be written as

$$
\left[\begin{array}{ll}
T & 0 \\
0 & 0
\end{array}\right]\left[\begin{array}{c}
\dot{\widetilde{P}} \\
\dot{\widetilde{\Lambda}}
\end{array}\right]=\left[\begin{array}{cc}
C & S^{T} \\
S & 0
\end{array}\right]\left[\begin{array}{c}
\widetilde{P} \\
\widetilde{\Lambda}
\end{array}\right]+\left[\begin{array}{l}
b \\
h
\end{array}\right]
$$

where $T=\operatorname{diag}\left\{\tau_{g 1}, \tau_{g 2}, \ldots, \tau_{g m}, \tau_{d 1}, \tau_{d 2}, \ldots, \tau_{d n}\right\}, \tau_{g i}>0$, $\tau_{d j}>0, C=\operatorname{diag}\left\{-c_{g 1},-c_{g 2}, \ldots,-c_{g m}, c_{d 1}, c_{d 2}, \ldots c_{d n}\right\}, \widetilde{\Lambda}=$ $\left[\begin{array}{llll}\lambda & \mu_{1} & \cdots & \mu_{n_{s}}\end{array}\right], i=1,2, \ldots, m, j=1,2, \ldots, n, \widetilde{P} \in R^{m+n}$, $\widetilde{\Lambda} \in R^{n_{S}+1}$, and $\mu_{k}$ is the Lagrange multiplier for $k=1,2, \ldots$, $n_{S}$. The matrix $S$ corresponds to the sensitivities of the constraints:

$$
\left[\begin{array}{cccccc}
1 & \cdots & 1 & -1 & \cdots & -1 \\
s_{g 11} & \cdots & s_{g 1 m} & s_{d 11} & \cdots & s_{d 1 n} \\
\vdots & & \vdots & \vdots & & \vdots \\
s_{g n_{S} 1} & \cdots & s_{g n_{S} m} & s_{d n_{S} 1} & \cdots & s_{d n_{S} n}
\end{array}\right]
$$

where the first row represents the power balance condition; $b=\left[\begin{array}{llllllll}-b_{g 1} & -b_{g 2} & \cdots & -b_{g m} & b_{d 1} & b_{d 2} & \cdots & b_{d n}\end{array}\right]^{T}$ is a cost vector of linear coefficients; $h=\left[\begin{array}{lllll}0 & h_{1} & h_{2} & \cdots & h_{n_{S}}\end{array}\right]^{T}$ is a vector with the value of the fixed demand in its first position and the values of the right hand sides in the constraint equations in the remaining positions. 
Because there is at least an equilibrium point in this system, through the translation transformation, it can be denoted as follows:

$$
\left[\begin{array}{ll}
T & 0 \\
0 & 0
\end{array}\right]\left[\begin{array}{l}
\dot{P} \\
\dot{\Lambda}
\end{array}\right]=\left[\begin{array}{cc}
C & S^{T} \\
S & 0
\end{array}\right]\left[\begin{array}{l}
P \\
\Lambda
\end{array}\right]
$$

2.2. The Electricity Market Interval Model. In power market, due to the influence of the related factors such as measurement error, technical level, and other external interference, the accurate values of some parameters cannot be obtained. Therefore, interval theory should be introduced to describe problems more appropriately.

There is the definition of interval matrix in the theory of differential equations. If $\underline{A}=\left(u_{i j}\right)_{m \times n}$ and $\bar{A}=\left(v_{i j}\right)_{m \times n}$ are two matrices with property in which $u_{i j} \leq v_{i j}$ for any $1 \leq i \leq$ $m$ and $1 \leq j \leq n$, the $m \times n$ interval matrix $[\underline{A}, \bar{A}]$ is defined by

$$
\begin{aligned}
& {[\underline{A}, \bar{A}]} \\
& \quad=\left\{A=\left(a_{i j}\right)_{m \times n}: u_{i j} \leq a_{i j} \leq v_{i j}, 1 \leq i \leq m, 1 \leq j \leq n\right\} .
\end{aligned}
$$

Due to the influence of dynamic factors in electricity market like economic growth, electricity consumption, electricity price, supply, and demand, the demand elasticity of suppliers and consumers continues to change within a certain range instead of constants. In order to describe the power market operation more accurately, it is necessary to set up a power market model with demand elasticity interval for suppliers and consumers, corresponding to the diagonal elements of diagonal matrix $C$ within a certain interval in (6). For coefficient matrix $C$, the range of each element is defined by $\underline{c}_{g i}<c_{g i}<\bar{c}_{g i}$ and $\underline{c}_{d j}<c_{d j}<\bar{c}_{d j}$. It is signified that $C_{I}=[\underline{C}, \bar{C}]$, where

$$
\begin{aligned}
& \underline{C}=\operatorname{diag}\left\{-\bar{c}_{g 1},-\bar{c}_{g 2}, \ldots,-\bar{c}_{g m}, \underline{c}_{d 1}, \underline{c}_{d 2}, \ldots, \underline{c}_{d n}\right\}, \\
& \bar{C}=\operatorname{diag}\left\{-\underline{c}_{g 1},-\underline{c}_{g 2}, \ldots,-\underline{c}_{g m}, \bar{c}_{d 1}, \bar{c}_{d 2}, \ldots, \bar{c}_{d n}\right\} .
\end{aligned}
$$

Usually, there exists $m+n>n_{S}+1$. Let $\operatorname{rank}(S)=n_{S}+1$ and $S=\left(S_{1}, S_{2}\right)$, where $S_{1}$ corresponds to a $\left(n_{S}+1\right) \times\left(n_{S}+1\right)$ nonsingular submatrix of $S$. The matrices $T$ and $C_{I}$ can be divided into the following form:

$$
T=\left[\begin{array}{cc}
T_{1} & 0 \\
0 & T_{2}
\end{array}\right], \quad C_{I}=\left[\begin{array}{cc}
C_{1 I} & 0 \\
0 & C_{2 I}
\end{array}\right],
$$

where $T_{1}$ and $C_{1 I}$ are $\left(n_{S}+1\right) \times\left(n_{S}+1\right)$ diagonal matrices; $T_{2}$ and $C_{2 I}$ are $q \times q$ diagonal matrices, and $q=(m+n)-\left(n_{S}+1\right)$. In a similar definition to the matrix $C_{I}, C_{1 I}$ and $C_{2 I}$ are interval matrices $C_{1 I}=\left[\underline{C}_{1}, \bar{C}_{1}\right]$ and $C_{2 I}=\left[\underline{C}_{2}, \bar{C}_{2}\right]$, respectively. Obviously, $C_{1} \in C_{1 I}$ and $C_{2} \in C_{2 I}$.

Therefore, the electricity market dynamic model (8) can be improved by the following interval dynamic system model:

$$
\left[\begin{array}{ccc}
T_{1} & 0 & 0 \\
0 & T_{2} & 0 \\
0 & 0 & 0
\end{array}\right]\left[\begin{array}{l}
\dot{P}_{1} \\
\dot{P}_{2} \\
\dot{\Lambda}
\end{array}\right]=\left[\begin{array}{ccc}
C_{1 I} & 0 & S_{1}^{T} \\
0 & C_{2 I} & S_{2}^{T} \\
S_{1} & S_{2} & 0
\end{array}\right]\left[\begin{array}{c}
P_{1} \\
P_{2} \\
\Lambda
\end{array}\right]
$$

Since the matrix $S_{1}$ is nonsingular, reduction and elimination to $P_{1}$ yield the purely reduced differential equation as follows:

$$
-T_{1} S_{1}^{-1} S_{2} \dot{P}_{2}=-C_{1 I} S_{1}^{-1} S_{2} P_{2}+S_{1}^{T} \Lambda
$$

Obtaining $\Lambda$ from (13) and substituting it into the second group equation of (12), we can rewrite (12) as follows:

$$
\left(T_{2}+S_{3}^{T} T_{1} S_{3}\right) \dot{P}_{2}=\left(C_{2 I}+S_{3}^{T} C_{1 I} S_{3}\right) P_{2},
$$

where $S_{3}=S_{1}^{-1} S_{2}$.

Let $T_{3}=T_{2}+S_{3}^{T} T_{1} S_{3}$; system (14) could be written as the following interval dynamical system in electric power market:

$$
T_{3} d P_{2}(t)=\left(C_{2 I}+S_{3}^{T} C_{1 I} S_{3}\right) P_{2}(t) d t
$$

\section{The Interval Stability Theorems}

In this section, we consider the stability of interval dynamical system (15) in electric power market. Let $|\cdot|$ denote the Euclidean norm of a column vector in $R^{n}$. If $A$ is a vector or matrix, its transpose is denoted by $A^{T}$. If $A$ is a matrix, its operator norm is denoted by $\|A\|$. If $A$ is a symmetric matrix, $\lambda_{\max }(A)$ and $\lambda_{\min }(A)$ are used to signify its largest and smallest eigenvalue, respectively.

According to the interval dynamical system theory of differential equations, let us consider the following system:

$$
d X(t)=A_{I} X(t) d t
$$

Definition 1 (see [19]). If the equilibrium solution of system (16) is asymptotically stable for any $A_{I} \in[\bar{A}, \underline{A}]$, then the system (16) is interval stable.

In [20], the differential operator $L$ is

$$
L=\frac{\partial}{\partial t}+\sum_{i=1}^{n} f_{i}(X, t) \frac{\partial}{\partial x_{i}}
$$

where $f(X, t)=A_{I} X(t)$.

Next, we give some preliminary lemmas, which play an important role in the proof of our stability theorem.

Lemma 2 (see [15]). Let $A_{n \times n}$ be a real positive semidefinite matrix and $S_{n \times r}$ a certain real matrix; then $B=S^{T} A S$ is a positive semidefinite matrix.

Lemma 3 (see [15]). The sum of positive definite matrix and positive semidefinite matrix will be positive definite matrix; the sum of negative definite matrix and negative semidefinite matrix will be negative definite matrix; the sum of two positive definite matrices will be positive definite matrix; the sum of two negative definite matrices will be negative definite matrix.

Lemma 4 (see [21]). If $A$ is a $n \times n$ real symmetric positive define matrix, then there must exist an invertible symmetric matrix $B$ satisfying $A=B^{2}$. 
Lemma 5 (see [21]). If $A$ is a $n \times n$ real symmetric matrix and $x$ is a -dimensional column vector, then

$$
\lambda_{\min }(A) x^{T} x \leq x^{T} A x \leq \lambda_{\max }(A) x^{T} x .
$$

Lemma 6 (see [22]). If there is a continuous positive definite function $V(t, x)$ on $I \times R^{n}$ such that $L V$ is negative definite and there exist infinitesimally upper bound and infinitely great lower bound for $V(t, x)$, then the system $d X(t)=A X(t) d t$ is asymptotically stable.

Since $T=\operatorname{diag}\left(\tau_{g 1}, \ldots, \tau_{g m}, \tau_{d 1}, \ldots, \tau_{d n}\right)=\operatorname{diag}\left(T_{1}, T_{2}\right)$, $\tau_{g i}>0, \tau_{d j}>0$, the matrices $T, T_{1}, T_{2}$ are positive definite. By Lemma 2, the matrix $S_{3}^{T} T_{3} S_{3}$ is positive semidefinite. Then, by Lemma 3, the matrix $T_{3}=T_{2}+S_{3}^{T} T_{1} S_{3}$ is symmetric positive definite and reversible.

Equation (15) can be represented as a common interval linear system:

$$
d P_{2}(t)=T_{3}^{-1}\left(C_{2 I}+S_{3}^{T} C_{1 I} S_{3}\right) P_{2}(t) d t
$$

Let $\widetilde{C}_{1}=(1 / 2)\left(\bar{C}_{1}-\underline{C}_{1}\right)$ and $\widetilde{C}_{2}=(1 / 2)\left(\bar{C}_{2}-\underline{C}_{2}\right)$, which indicates that all the elements of $\widetilde{C}_{1}$ and $\widetilde{C}_{2}$ are nonnegative. Taking $C_{3}=C_{2}+S_{3}^{T} C_{1} S_{3}, C_{1 I}=C_{1}+\Delta C_{1}$, and $C_{2 I}=C_{2}+\Delta C_{2}$, then we have $\Delta C_{1} \in\left[-\widetilde{C}_{1}, \widetilde{C}_{1}\right]$ and $\Delta C_{2} \in\left[-\widetilde{C}_{2}, \widetilde{C}_{2}\right]$.

Alternatively, due to Lemma 4 , there exists an invertible matrix $T_{33}$ such that $T_{3}=\left(T_{33}^{-1}\right)^{T} T_{33}^{-1}$. Substituting this into (15) yields that

$$
\left(T_{33}^{-1}\right)^{T} T_{33}^{-1} d P_{2}(t)=\left[C_{3}+\left(\Delta C_{2}+S_{3}^{T} \Delta C_{1} S_{3}\right)\right] P_{2}(t) d t .
$$

By $\left(T_{33}^{-1}\right)^{T}=\left(T_{33}^{T}\right)^{-1}$, we have

$$
T_{33}^{-1} d P_{2}(t)=T_{33}^{T}\left[C_{3}+\left(\Delta C_{2}+S_{3}^{T} \Delta C_{1} S_{3}\right)\right] T_{33} T_{33}^{-1} P_{2}(t) d t .
$$

Put $X(t)=T_{33}^{-1} P_{2}(t)$; then $d X(t)=T_{33}^{-1} d P_{2}(t)$. Equation (21) can be transformed into

$$
d X(t)=T_{33}^{T}\left[C_{3}+\left(\Delta C_{2}+S_{3}^{T} \Delta C_{1} S_{3}\right)\right] T_{33} X(t) d t .
$$

Theorem 7. Assume that there exists a real symmetric positive definite matrix $Q$ such that

$$
\begin{aligned}
& h_{1}\left|T_{33}^{-1} P_{2}\right|^{2} \leq\left(T_{33}^{-1} P_{2}\right)^{T} Q T_{33}^{-1} P_{2} \leq h_{2}\left|T_{33}^{-1} P_{2}\right|^{2}, \\
& \lambda_{\max }\left(Q^{1 / 2} T_{33}^{T} C_{3} T_{33} Q^{-1 / 2}+Q^{-1 / 2} T_{33}^{T} C_{3} T_{33} Q^{1 / 2}\right) \\
& \quad+2\left\|T_{33}\right\|^{2} \cdot\left(\left\|\widetilde{C}_{2}\right\|+\left\|\widetilde{C}_{1}\right\| \cdot\left\|S_{3}\right\|^{2}\right) \cdot \sqrt{\frac{\|Q\|}{h_{1}}}<0,
\end{aligned}
$$

where $h_{1}, h_{2}$ are positive constants; then the interval dynamical system (15) is interval stable.
Proof. Considering the interval linear system (22), we choose the following Lyapunov function: $V(t, X)=X^{T} Q X$. According to the condition $h_{1}\left|T_{33}^{-1} P_{2}\right|^{2} \leq\left(T_{33}^{-1} P_{2}\right)^{T} Q T_{33}^{-1} P_{2} \leq h_{2}\left|T_{33}^{-1} P_{2}\right|^{2}$, we get $h_{1}|X|^{2} \leq X^{T} Q X \leq h_{2}|X|^{2}$. Therefore, there exist infinitesimally upper bound and infinitely great lower bound for $V(t, X)$.

Owing to $(\partial / \partial t)\left(X^{T} Q X\right)=0$, applying the $L$-operator to (15), we obtain

$$
\begin{aligned}
L V & =\sum_{i=1}^{k} f_{i}(X, t) \frac{\partial}{\partial x_{i}}\left(X^{T} Q X\right)=2 X^{T} Q f(X, t) \\
& =2 X^{T} Q T_{33}^{T} C_{3} T_{33} X+2 X^{T} Q T_{33}^{T}\left(\Delta C_{2}+S_{3}^{T} \Delta C_{1} S_{3}\right) T_{33} X
\end{aligned}
$$

By Lemma 5, the first items on the right are yielded as follows:

$$
\begin{aligned}
& 2 X^{T} Q T_{33}^{T} C_{3} T_{33} X \\
& \begin{array}{l}
=X^{T}\left[Q ^ { 1 / 2 } \left(Q^{1 / 2} T_{33}^{T} C_{3} T_{33} Q^{-1 / 2}\right.\right. \\
\left.\left.\quad+Q^{-1 / 2} T_{33}^{T} C_{3} T_{33} Q^{1 / 2}\right) Q^{1 / 2}\right] X \\
=\left(Q^{1 / 2} X\right)^{T}\left(Q^{1 / 2} T_{33}^{T} C_{3} T_{33} Q^{-1 / 2}\right. \\
\left.\quad+Q^{-1 / 2} T_{33}^{T} C_{3} T_{33} Q^{1 / 2}\right) Q^{1 / 2} X \\
\leq \lambda_{\max }\left(Q^{1 / 2} T_{33}^{T} C_{3} T_{33} Q^{-1 / 2}+Q^{-1 / 2} T_{33}^{T} C_{3} T_{33} Q^{1 / 2}\right) X^{T} Q X .
\end{array}
\end{aligned}
$$

As is well known, there is $2 a b \leq \varepsilon a^{2}+b^{2} / \varepsilon$ for any $\varepsilon>0$. Hence, the second item on the right becomes

$$
\begin{array}{rl}
2 X^{T} & Q T_{33}^{T}\left(\Delta C_{2}+S_{3}^{T} \Delta C_{1} S_{3}\right) T_{33} X \\
\leq & 2\left|X^{T} Q^{1 / 2}\right|\left|Q^{1 / 2} T_{33}^{T}\left(\Delta C_{2}+S_{3}^{T} \Delta C_{1} S_{3}\right) T_{33} X\right| \\
\leq & \varepsilon\left|X^{T} Q^{1 / 2}\right|^{2}+\frac{1}{\varepsilon}\left|Q^{1 / 2} T_{33}^{T}\left(\Delta C_{2}+S_{3}^{T} \Delta C_{1} S_{3}\right) T_{33} X\right|^{2} \\
= & \varepsilon X^{T} Q X+\frac{1}{\varepsilon} X^{T} T_{33}^{T}\left(\Delta C_{2}+S_{3}^{T} \Delta C_{1} S_{3}\right) T_{33} Q T_{33}^{T} \\
& \times\left(\Delta C_{2}+S_{3}^{T} \Delta C_{1} S_{3}\right) T_{33} X \\
\leq & \varepsilon X^{T} Q X+\frac{1}{\varepsilon}\|Q\| \cdot\left\|T_{33}\right\|^{4} \cdot\left\|\left(\Delta C_{2}+S_{3}^{T} \Delta C_{1} S_{3}\right)\right\|^{2} \cdot|X|^{2} .
\end{array}
$$

Using the properties of matrix norm, we can conclude

$$
\begin{aligned}
\left\|\left(\Delta C_{2}+S_{3}^{T} \Delta C_{1} S_{3}\right)\right\| & \leq\left\|\Delta C_{2}\right\|+\left\|\Delta C_{1}\right\| \cdot\left\|S_{3}\right\|^{2} \\
& \leq\left\|\widetilde{C}_{2}\right\|+\left\|\widetilde{C}_{1}\right\| \cdot\left\|S_{3}\right\|^{2} .
\end{aligned}
$$


The condition $h_{1}|X|^{2} \leq X^{T} Q X$ can be turned into $|X|^{2} \leq$ $X^{T} Q X / h_{1}$, putting it into (26) to get

$$
\begin{array}{rl}
2 X^{T} & Q T_{33}^{T}\left(\Delta C_{2}+S_{3}^{T} \Delta C_{1} S_{3}\right) T_{33} X \\
& \leq\left(\varepsilon+\frac{\|Q\| \cdot\left\|T_{33}\right\|^{4} \cdot\left(\left\|\widetilde{C}_{2}\right\|+\left\|\widetilde{C}_{1}\right\| \cdot\left\|S_{3}\right\|^{2}\right)^{2}}{\varepsilon \cdot h_{1}}\right) X^{T} Q X .
\end{array}
$$
hence,

Choosing $\varepsilon=\left\|T_{33}\right\|^{2} \cdot\left(\left\|\widetilde{C}_{2}\right\|+\left\|\widetilde{C}_{1}\right\| \cdot\left\|S_{3}\right\|^{2}\right) \cdot \sqrt{\|Q\| / h_{1}}$,

$$
\begin{aligned}
& 2 X^{T} Q_{33}^{T}\left(\Delta C_{2}+S_{3}^{T} \Delta C_{1} S_{3}\right) T_{33} X \\
& \quad \leq 2\left\|T_{33}\right\|^{2} \cdot\left(\left\|\widetilde{C}_{2}\right\|+\left\|\widetilde{C}_{1}\right\| \cdot\left\|S_{3}\right\|^{2}\right) \cdot \sqrt{\frac{\|Q\|}{h_{1}}} \cdot X^{T} Q X .
\end{aligned}
$$

Substituting (25) and (29) into (24), it can be yielded as

$$
\begin{aligned}
L V \leq & {\left[\lambda_{\max }\left(Q^{1 / 2} T_{33}^{T} C_{3} T_{33} Q^{-1 / 2}+Q^{-1 / 2} T_{33}^{T} C_{3} T_{33} Q^{1 / 2}\right)\right.} \\
& \left.+2\left\|T_{33}\right\|^{2} \cdot\left(\left\|\widetilde{C}_{2}\right\|+\left\|\widetilde{C}_{1}\right\| \cdot\left\|S_{3}\right\|^{2}\right) \cdot \sqrt{\frac{\|Q\|}{h_{1}}}\right] X^{T} Q X .
\end{aligned}
$$

When

$$
\begin{gathered}
\lambda_{\max }\left(Q^{1 / 2} T_{33}^{T} C_{3} T_{33} Q^{-1 / 2}+Q^{-1 / 2} T_{33}^{T} C_{3} T_{33} Q^{1 / 2}\right) \\
+2\left\|T_{33}\right\|^{2} \cdot\left(\left\|\widetilde{C}_{2}\right\|+\left\|\widetilde{C}_{1}\right\| \cdot\left\|S_{3}\right\|^{2}\right) \cdot \sqrt{\frac{\|Q\|}{h_{1}}}<0
\end{gathered}
$$

there exists $h_{3}>0$ such that $L V<-h_{3} \cdot V$, so $L V$ is negative definite. Summing up the above, the interval dynamical system (15) is asymptotically stable for any $C_{1} \in C_{1 I}$ and $C_{2} \in$ $C_{2 I}$ by Lemma 6 . According to the definition, the interval dynamical system (15) is interval stable.

For the sake of simplicity, in particular, let $Q=E$; choosing $h_{1}=h_{2}=1$, then the following corollary can be concluded.

Corollary 8. If $\lambda_{\max }\left(T_{33}^{T} C_{3} T_{33}\right)+\left(\left\|\widetilde{C}_{2}\right\|+\left\|\widetilde{C}_{1}\right\| \cdot\left\|S_{3}\right\|^{2}\right)\left\|T_{33}\right\|^{2}$ $<0$, then the interval dynamical system (15) in electricity market is interval stable.

Targeted to the interval dynamical system in electricity market, the corollary puts forward a concise decision condition for analyzing the stability of this power system. It is indicated that we can judge the system stability by available data and provide the stability scopes of demand elasticity for suppliers and consumers. Next, we will illustrate it through a specific example below.

\section{The Numerical Example}

In this section, we will use the theorem of interval stability to analyze specific interval model (15) in electricity market, verifying the validity of the theorem. Linear system (19) is the equivalent deformation of interval dynamical system (15). In order to make the result more clear and facilitate data analysis and numerical simulation for power market interval model, the following will make a case analysis in power market dynamic system (19).

The original data comes from Table 4 in literature [2]. Corresponding to the congestion condition $n_{S}=2$ of three suppliers and two consumers, the demand elasticity of each supplier is $0.3,0.5$, and 0.2 and response speeds of generation power output are $0.1,0.3$, and 0.2 , respectively; the demand elasticity of each consumer is -0.5 and -0.6 and expansion speeds of each consumer demand are 0.2 and 0.25 , respectively; the steady-state values of each electricity demand are 7.68 and 8.05 , respectively. Consider

$$
S_{1}=\left[\begin{array}{ccc}
1 & 1 & 1 \\
0.1 & -0.1 & 0 \\
0.2 & 0 & 0.3
\end{array}\right], \quad S_{2}=\left[\begin{array}{cc}
-1 & -1 \\
0.1 & -0.1 \\
-0.1 & -0.1
\end{array}\right] \text {. }
$$

Based on these data, there are

$$
\begin{gathered}
C_{1}=\operatorname{diag}\{-0.3,-0.5,-0.2\}, \quad C_{2}=\operatorname{diag}\{-0.5,-0.6\}, \\
T_{3}=[0.425,-0.025 ;-0.025,0.475] \\
S_{3}=[0.25,-1.25 ;-0.75,-0.25 ;-0.5,0.5] \\
P_{2}(t)=\left[P_{d 1}(t), P_{d 2}(t)\right]^{T}
\end{gathered}
$$

Using the corollary above, we can calculate the detailed range of demand elasticity for suppliers and consumers as follows:

$$
\begin{array}{cc}
0.07<c_{g 1}<0.53 ; & 0.29<c_{g 2}<0.71 ; \\
-0.03<c_{g 3}<0.43 ; & -0.85<c_{d 1}<-0.15 ; \\
-0.961<c_{d 2}<-0.239 .
\end{array}
$$

Therefore,

$\widetilde{C}_{1}=\operatorname{diag}\{0.23,0.21,0.23\}, \quad \widetilde{C}_{2}=\operatorname{diag}\{0.35,0.361\}$.

We compute

$$
\begin{aligned}
& \lambda_{\max }\left(T_{33}^{T} C_{3} T_{33}\right)+\left(\left\|\widetilde{C}_{2}\right\|+\left\|\widetilde{C}_{1}\right\| \cdot\left\|S_{3}\right\|^{2}\right)\left\|T_{33}\right\|^{2} \\
& =-0.0113<0,
\end{aligned}
$$

fulfilling the conditions of the corollary, so this system is interval stable, which means that the system is stable as long as the demands elasticity range within the given interval. So as to illustrate the validity of the theoretical analysis, we select four different typical sets of data about the demand elasticity of suppliers and consumers. The relevant coefficient matrices and figures of each set in system (19) are computed and simulated by MATLAB. The details about the results are shown in Table 1 and Figures 1, 2, 3, and 4. 
TABLE 1: The eigenvalues of coefficient matrices with different parameters.

\begin{tabular}{cccccccrr}
\hline$c_{g 1}$ & $c_{g 2}$ & $c_{g 3}$ & $c_{d 1}$ & $c_{d 2}$ & Coefficient matrix & $\lambda_{1}$ & $\lambda_{2}$ & Figure \\
\hline 0.07 & 0.29 & -0.03 & -0.85 & -0.961 & {$\left[\begin{array}{ll}-0.737 & -0.139 \\
-0.123 & -0.763\end{array}\right]$} & -0.881 & -0.618 & Figure 1 \\
\hline 0.30 & 0.50 & 0.20 & -0.50 & -0.60 & {$\left[\begin{array}{rrr}-0.200 & -0.025 \\
0.000 & -2.422\end{array}\right]$} & -2.422 & -2.000 & Figure 2 \\
\hline 0.13 & 0.63 & -0.01 & -0.75 & -0.32 & {$\left[\begin{array}{lll}-2.630 & -0.258 \\
-0.307 & -1.193\end{array}\right]$} & -2.683 & -1.140 & Figure 3 \\
\hline 0.53 & 0.71 & 0.43 & -0.15 & -0.239 & {$\left[\begin{array}{llll}-3.263 & -0.089 \\
0.123 & -4.082\end{array}\right]$} & -4.095 & -3.250 & Figure 4 \\
\hline
\end{tabular}

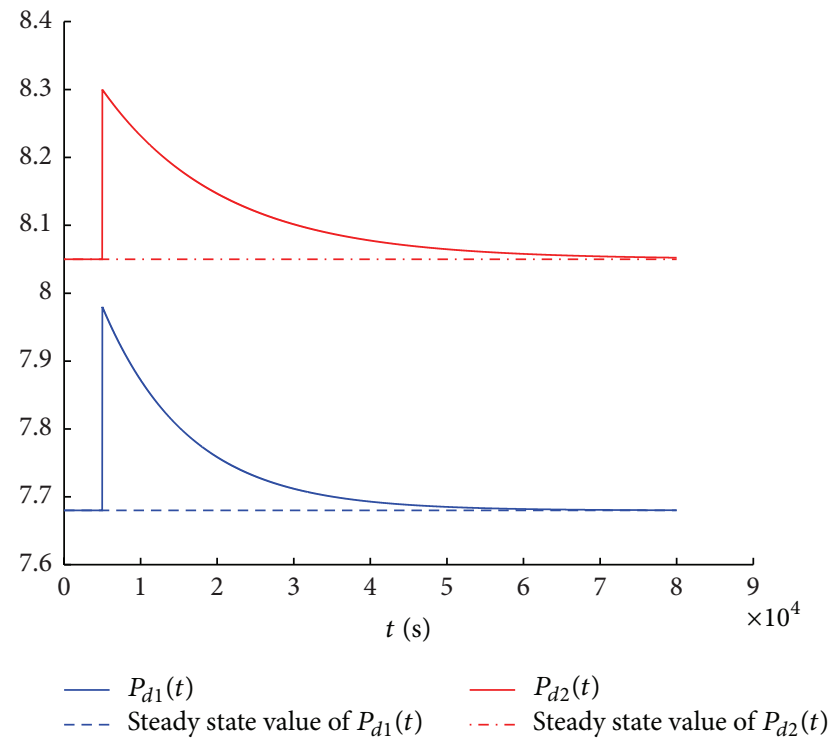

Figure 1: Value change of $P_{d 1}$ and $P_{d 2}$ with $\Delta C_{1}=\operatorname{diag}\{0.23,0.21$, $0.23\}$ and $\Delta C_{2}=\operatorname{diag}\{-0.35,-0.361\}$.

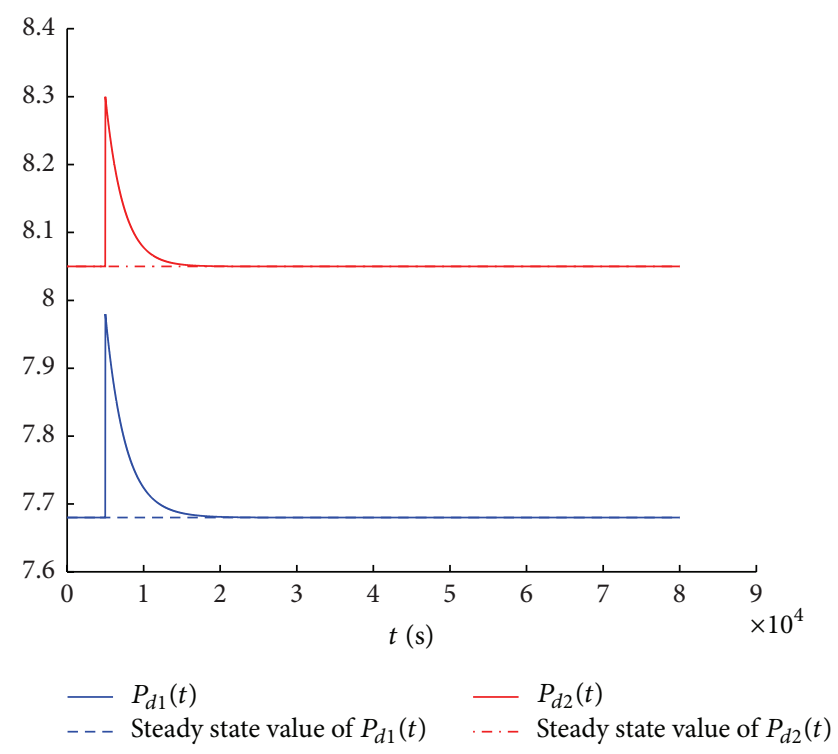

FIgURE 2: Value change of $P_{d 1}$ and $P_{d 2}$ with $\Delta C_{1}=\operatorname{diag}\{0,0,0\}$ and $\Delta C_{2}=\operatorname{diag}\{0,0\}$.

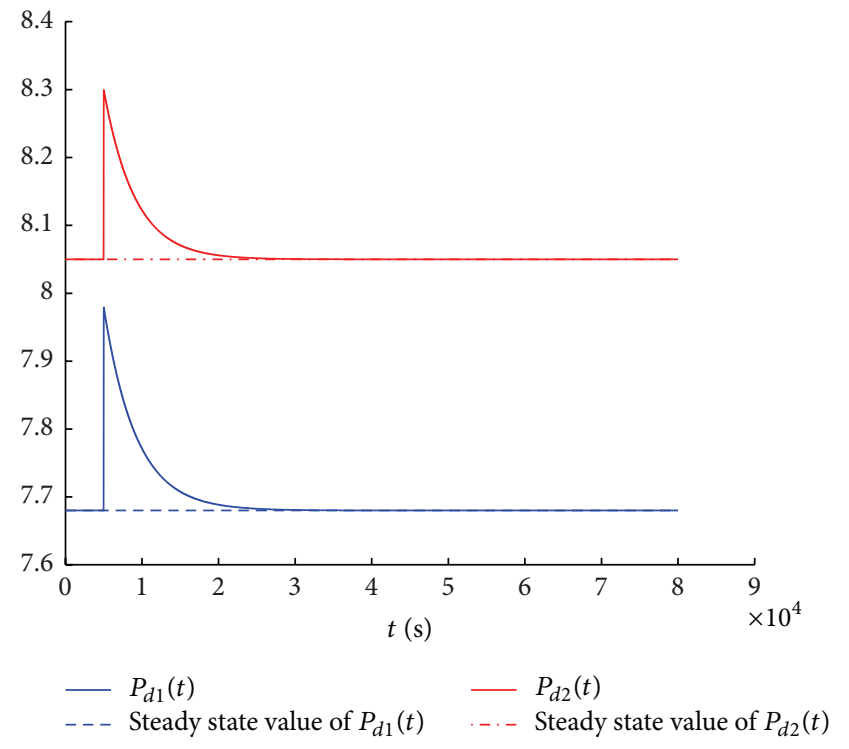

FIgUre 3: Value change of $P_{d 1}$ and $P_{d 2}$ with $\Delta C_{1}=\operatorname{diag}\{0.17,0.13$, $0.21\}$ and $\Delta C_{2}=\operatorname{diag}\{-0.25,0.28\}$.

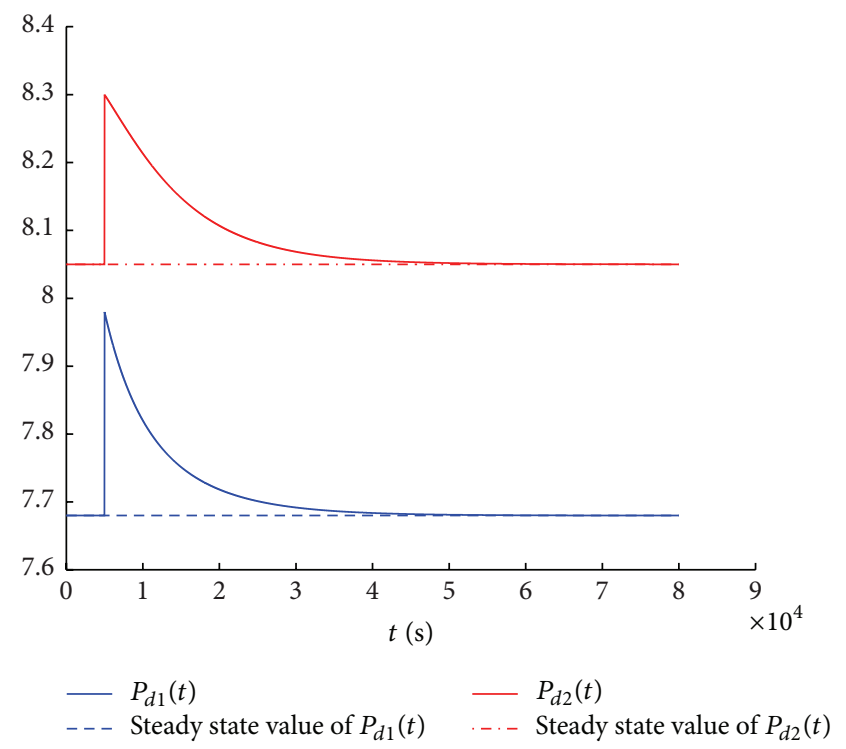

FIGURE 4: Value change of $P_{d 1}$ and $P_{d 2}$ with $\Delta C_{1}=\operatorname{diag}\{-0.23,-0.21$, $-0.23\}$ and $\Delta C_{2}=\operatorname{diag}\{0.35,0.361\}$. 
The eigenvalues of the coefficient matrix with different demand elasticity are provided in Table 1, including the endpoints and interior points of demand elasticity interval. The accurate change intervals are $\Delta C_{1}=\operatorname{diag}\{0.23,0.21$, $0.23\}, \Delta C_{2}=\operatorname{diag}\{-0.35,-0.361\} ; \Delta C_{1}=\operatorname{diag}\{0,0,0\}, \Delta C_{2}=$ $\operatorname{diag}\{0,0\} ; \Delta C_{1}=\operatorname{diag}\{0.17,0.13,0.21\}, \Delta C_{2}=\operatorname{diag}\{-0.25$, $0.28\} ; \Delta C_{1}=\operatorname{diag}\{-0.23,-0.21,-0.23\}, \Delta C_{2}=\operatorname{diag}\{0.35$, $0.361\}$, respectively.

It can be turned out that the eigenvalues of the coefficient matrix are negative so long as the values of demand elasticity are in the interval, which means that the system is always stable in electricity market on this condition. It can be seen that all the values of $P_{2}(t)$ tend to be stable over time in Figures 1 to 4 , that is to say, the trends of electricity demand toward steady-state values, accounting for the asymptotic stability of system (19). The calculation results are consistent with the theoretical analysis above, which explains and verifies the effectiveness of the theorem and corollary.

\section{Conclusions}

Based on the electricity market dynamic model proposed by Alvarado, an interval dynamical system model is set up in power market under the consideration that the demand elasticity of suppliers and consumers continues to change within a certain range instead of constants. Targeted to the interval model, the sufficient determined conditions of interval stability are derived by constructing a suitable Lyapunov function and using the theory of interval dynamical system in differential equations and matrix inequality theory and so forth. It is concluded that the conditions can judge the system stability by available data about demand elasticity of suppliers and demands. Finally, the results of numerical examples are consistent with the stability analysis. The numerical simulation illustrates that the determined conditions of stability are effective, practical, and advantageous. The research method of this paper is expected to analyze the stability of power market with random disturbances in future.

\section{Conflict of Interests}

The authors declare that there is no conflict of interests regarding the publication of this paper.

\section{Acknowledgments}

This work was jointly supported by the National Natural Science Foundation of China (51190103 and 61374080) and a Project Funded by the Priority Academic Program Development of Jiangsu Higher Education Institutions.

\section{References}

[1] B. Beavis and I. M. Dobbs, Optimisation and Stability Theory for Economic Analysis, Cambridge University Press, Cambridge, UK, 1990

[2] F. Alvarado, “The stability of power system markets," IEEE Transactions on Power Systems, vol. 14, no. 2, pp. 505-511, 1999.
[3] F. L. Alvarado, J. Meng, W. S. Mota, and C. L. DeMarco, "Dynamic coupling between power markets and power systems," in Proceedings of the Power Engineering Society Summer Meeting, pp. 2201-2205, IEEE, July 2000.

[4] F. L. Alvarado and W. S. Mota, "The role of energy imbalance management on power market stability," in Proceedings of the 31st Annual Hawaii International Conference on System Sciences (HICSS '98), vol. 3, IEEE Computer Society, 1998.

[5] W. S. Mota and F. L. Alvarado, "Power markets stability considering energy imbalance," in Proceedings of the IEEE Internaitonal Symposium on Circuits and Systems, pp. V-665V-668, Geneva, Switzerland, May 2000.

[6] D. H. M. Nguyen and K. P. Wong, "A general stability metric for competitive power markets," in Electric Utility Deregulation and Restructuring and Power Technologies, 2000.

[7] Y. Xue, "Interactions between power market stability and power system stability," Automation of Electric Power Systems, vol. 26, no. 21, pp. 1-6, 2003.

[8] J. Shang, Y. Huang, and Q. Xia, Research on Electricity Market Theory and Its Applications, 2002.

[9] Z.-F. Tan, G.-Q. Tang, E.-K. Yu, and Y. Li, "Power market dynamic analysis," Proceedings of the Chinese Society of Electrical Engineering, vol. 21, no. 12, pp. 88-92, 2001.

[10] Y.-H. Hou and F. L. Wu, "Stability of electricity market with cyclic load demand," Proceedings of the Chinese Society of Electrical Engineering, vol. 26, no. 24, pp. 12-17, 2006.

[11] J. Nutaro and V. Protopopescu, "The impact of market clearing time and price signal delay on the stability of electric power markets," IEEE Transactions on Power Systems, vol. 24, no. 3, pp. 1337-1345, 2009.

[12] D. Watts and F. L. Alvarado, "The influence of futures markets on real time price stabilization in electricity markets," in Proceedings of the 37th Hawaii International Conference on System Sciences (HICSS '04), pp. 757-764, IEEE, January 2004.

[13] M. Bask and A. Widerberg, "Market structure and the stability and volatility of electricity prices," Energy Economics, vol. 31, no. 2, pp. 278-288, 2009.

[14] S. K. M. Kodsi, Accounting for the Effects of Power System Controllers and Stability on Power Dispatch and Electricity Market Prices, University of Waterloo, 2005.

[15] Z. H. Lu, G. Y. Li, and M. Zhou, "Study of electricity market stability model," in Proceedings of the 7th IET International Conference on Advances in Power System Control, Operation and Management (APSCOM '06), p. 369, Hong Kong, 2006.

[16] X. X. Liao and X. Mao, "Exponential stability of stochastic delay interval systems," Systems \& Control Letters, vol. 40, no. 3, pp. 171-181, 2000.

[17] C. Hua, X. Guan, and P. Shi, "Robust stabilization of a class of nonlinear time-delay systems," Applied Mathematics and Computation, vol. 155, no. 3, pp. 737-752, 2004.

[18] C. H. Su and S. F. Liu, "Mean-square robust stability of uncertain stochastic linear systems with time-varying interval parameters," Journal of Systems Science and Mathematical Sciences, vol. 30, no. 3, pp. 289-295, 2010.

[19] X.-X. Liao and J.-L. Qian, "Some new results for stability of interval matrices," Control Theory and Advanced Technology, vol. 7, pp. 265-275, 1988.

[20] C. Rakpenthai, S. Uatrongjit, and S. Premrudeeprechacharn, "State estimation of power system considering network parameter uncertainty based on parametric interval linear systems," IEEE Transactions on Power Systems, vol. 27, no. 1, pp. 305-313, 2012. 
[21] S. G. Wang, M. X. Wu, and Z. Z. Jia, Matrix Inequality, Science Press, Beijing, China, 2006.

[22] Z. E. Ma and Y. C. Zhou, Qualitative and Stability for Ordinary Differential Equations, Science Press, 2005. 


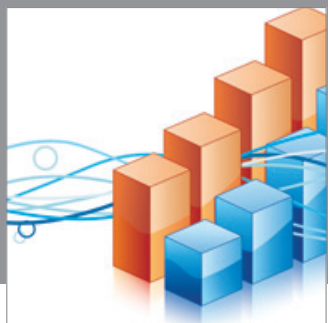

Advances in

Operations Research

mansans

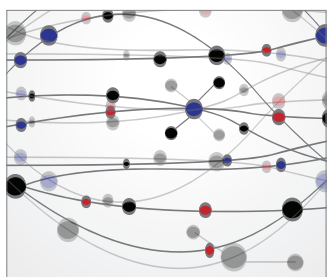

The Scientific World Journal
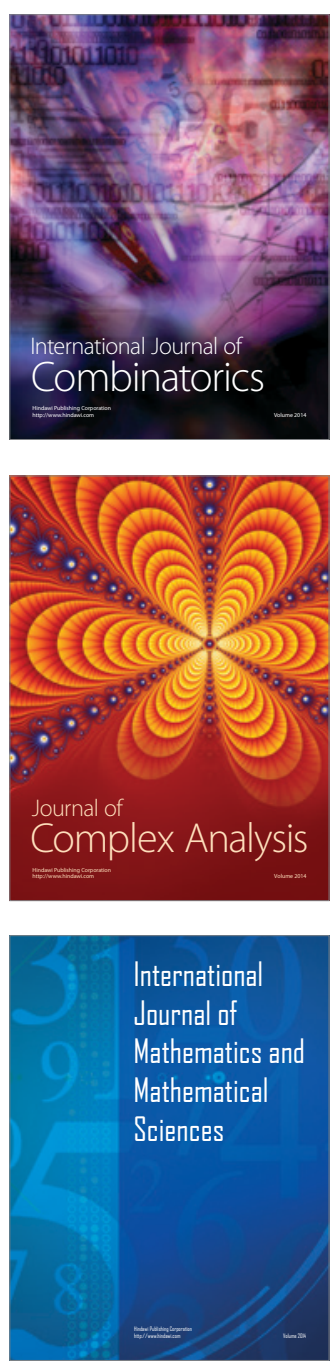
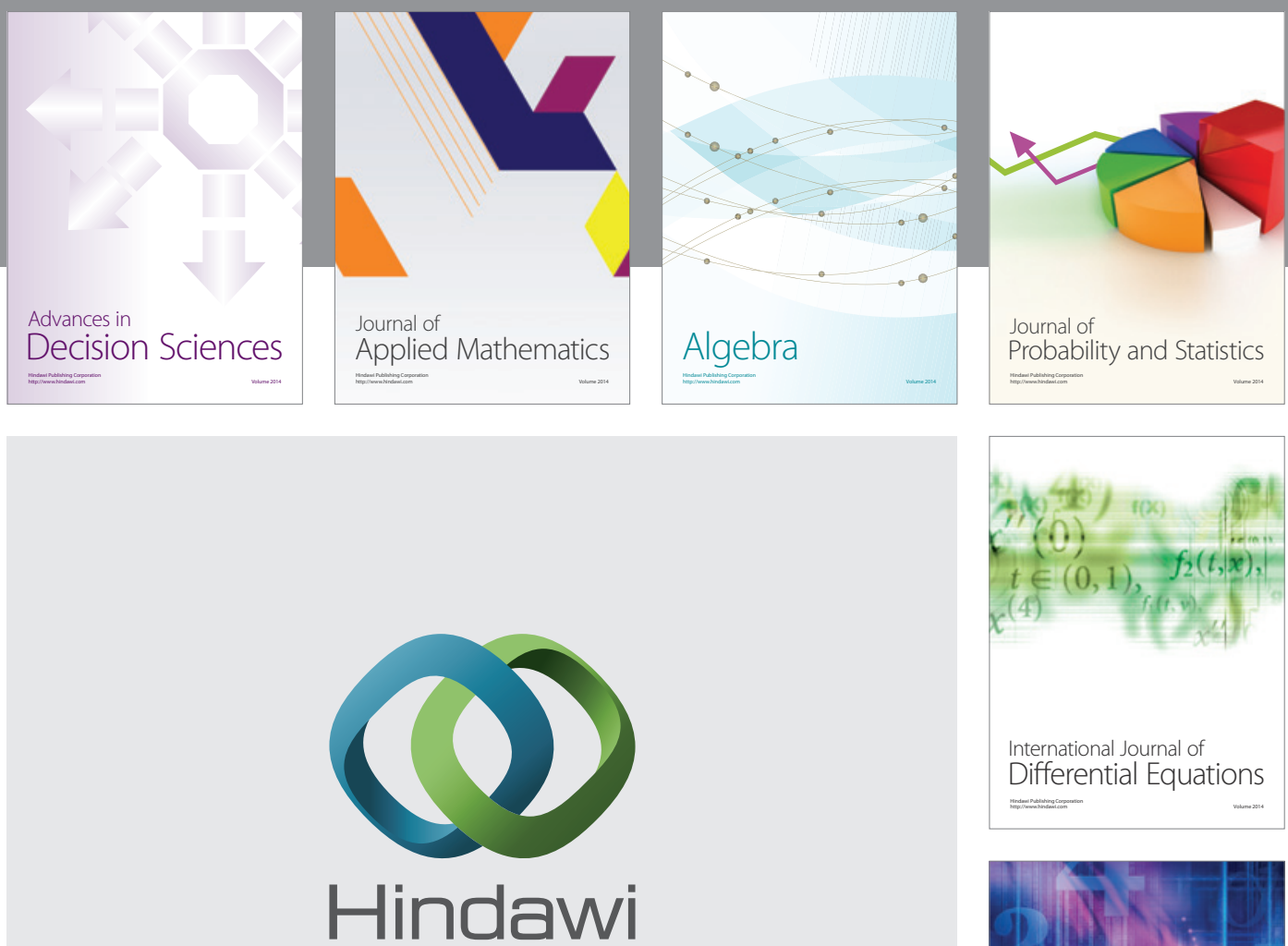

Submit your manuscripts at http://www.hindawi.com
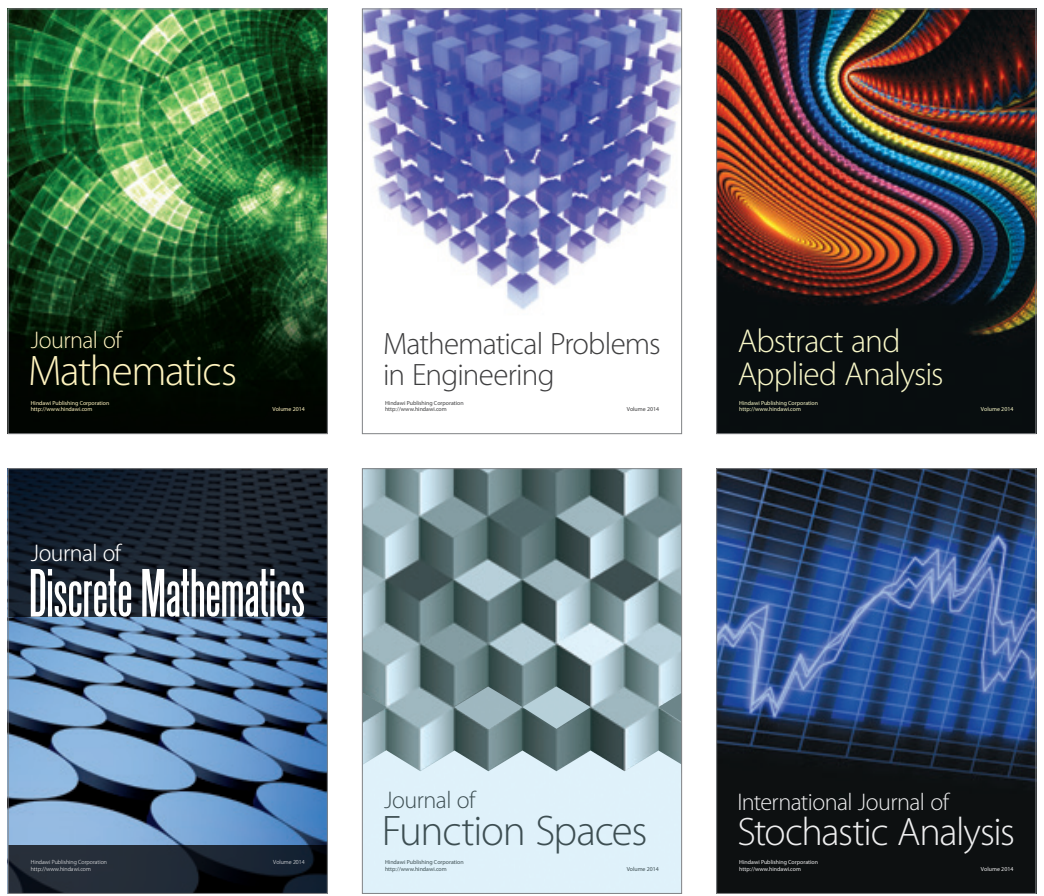

Journal of

Function Spaces

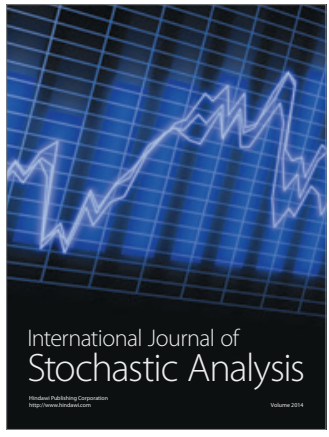

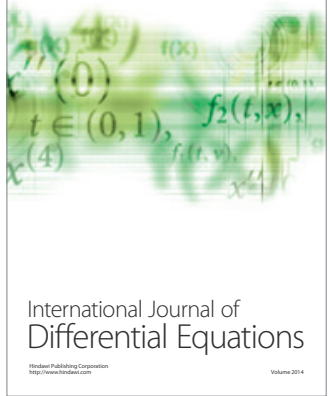
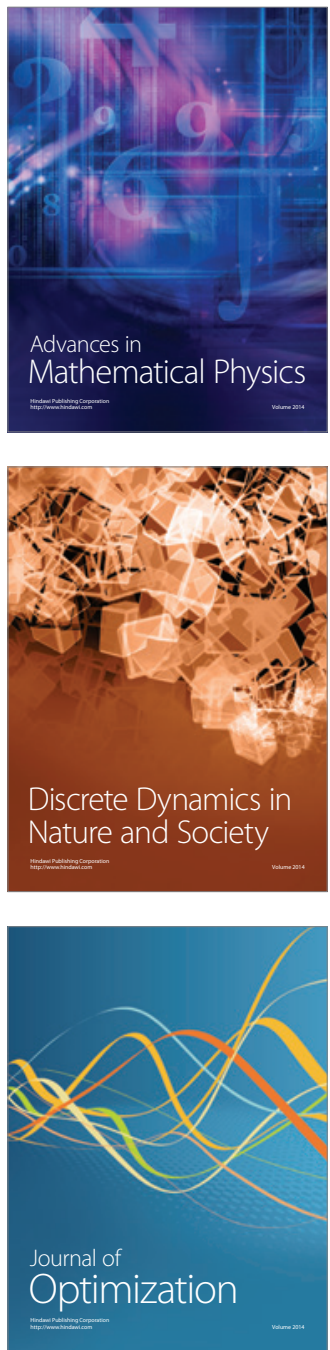